\title{
X-ray resonant powder diffraction
}

\author{
H. Palancher ${ }^{1, a}$, S. Bos ${ }^{2}$, J.F. Bérar ${ }^{2}$, I. Margiolaki ${ }^{3,4}$, and J.L. Hodeau ${ }^{2}$ \\ 1 CEA, DEN, DEC, 13108 Saint-Paul-Lez-Durance Cedex, France \\ 2 Institut Neel - CNRS / UJF, 25 rue des Martyrs, BP. 166, 38042 Grenoble Cedex 09, \\ France \\ 3 ESRF, 6 rue Horowitz, BP. 220, 38043 Grenoble, France \\ 4 Department of Biology, Section of Genetics, Cell Biology and Development, University of \\ Patras, 26500 Patras, Greece
}

Received 23 December 2011 / Received in final form 23 March 2012 Published online 15 June 2012

\begin{abstract}
X-ray resonant diffraction can be applied in structural chemistry studies on powder samples. It enables an important limitation of powder diffraction to be overcome. This limitation is related to the low ability of powder diffraction to differentiate elements with close atomic numbers when they occupy the same or close crystallographic sites (mixed occupancy case) and also to discriminate cations with different valence states in different sites. However the resonant effect usually has a second order influence on the measured intensity. As a consequence, the efficiency of this method directly implies the need for excellent quality data collection and has generally been better assessed on elements present in single phase powder samples. In recent years, instrumental developments have been made in synchrotron radiation facilities which allow easier use of resonant powder diffraction for site-specific contrast and valence i.e. oxidation state analyses. Moreover, resonant contrast diffraction tools also have been proposed for better visualization of the anomalous effect both in direct and reciprocal space by using differences between electron density maps or diffraction patterns. Finally the potentialities of this technique for de novo structure solution on macromolecular systems are mentioned.
\end{abstract}

\section{Introduction}

Structural research using resonant scattering (so-called anomalous diffraction) started a long time ago: as far back as 1930 this effect has been used for investigating the stacking sequence of atoms in sphalerite [1] or for discriminating-atoms of nearly equal atomic number [2-4]. In 1949 Bijvoet showed that we can take advantage of this effect to address the absolute configuration issue, thus opening the way to one of the major application of resonant (or anomalous) dispersion [5]: determination of the absolute configuration for chiral molecules. In the same paper, Bijvoet also pointed out the interest of resonant diffraction to solve the phase problem for structure factor determination. However, since other methods like the "Patterson method" or the

\footnotetext{
a e-mail: herve.palancher@cea.fr
} 
"direct method" were successful to solve small crystal structures, the first important use of resonant diffraction was for very large molecular systems. Then in the nineteen eighties-nineties X-ray resonant dispersion was used for MAD Multiwavelength Anomalous Diffraction (MAD) experiments in bio-macromolecular crystallography [6-8]. However all these experiments have been performed on single crystals.

In the early nineteen nineties, the wide availability of tunable and intense X-ray synchrotron facilities favored the development of resonant scattering applications for a wide range of materials [9] as for example, alloys [10] or amorphous materials [11,12]. On powders, which is the scope of this communication, resonant scattering has been used to solve either mixed valence or mixed occupancy problems, first on case study samples and then very soon in applied materials like high $\mathrm{T}_{\mathrm{c}}$ superconductor [13] or zeolites [14]. Nowadays it can be considered that the efficiency of this method has been demonstrated on numerous materials [11,15-19].

In this paper, after having very briefly described the basics of resonant diffraction, the strength and limits of this technique will be evidenced. Using a few examples, we will focus first on charge order determination using site selective $f^{\prime}$ determination (mixed valence case) and then site order determination (mixed occupancy case). The last section of this work deals with the potentialities of MAD methods for solving de novo complex structures.

\section{Resonant effect on polycrystalline samples: Basics and limitations}

Let us consider the diffraction by X-rays of a single phase polycrystalline sample. The intensity of each ( $h \mathrm{kl}$ ) Bragg line is proportional to the square of the modulus of the so-called $F(\mathbf{Q}, E)$ structure factor. It can be described by:

$$
F(\mathrm{Q}, \mathrm{E})=\sum_{i} f_{\mathrm{i}}(\mathbf{Q}, \mathrm{E}) \cdot \exp \left(\mathrm{i} \mathbf{Q} \cdot \mathrm{r}_{\mathrm{i}}\right) \cdot \exp \left(-\mathrm{B}_{\mathrm{i}} \mathbf{Q}^{2}\right)
$$

Where $i$ refers to the different occupied crystallographic sites, Q is the scattering vector and $\mathrm{B}_{i}$ the disorder parameter. In this equation, the chemical selectivity is provided by the atomic scattering factor $f_{i}(\mathbf{Q}, E)$. It can be defined as the sum over three terms: the energy independent Thomson scattering $f_{i}^{0}$ and the complex energy dependent (resonant or anomalous) scattering $f_{i}^{\prime}(\mathrm{E})+\mathrm{i} f_{i}^{\prime \prime}(\mathrm{E})$ :

$$
f_{\mathrm{i}}(\mathbf{Q}, \mathrm{E})=f_{i}^{0}(\mathbf{Q})+f_{\mathrm{i}}(\mathrm{E})+\mathrm{i} f_{\mathrm{i}}^{\prime \prime}(\mathrm{E}) .
$$

As a general rule, the energy dependent scattering term is not a scalar but a tensor, whose symmetry is given by the symmetry of the crystallographic site $i$ [20-22] Causality implies that $f_{i}^{\prime}(\mathrm{E})$ (dispersion) and $f_{i}^{\prime \prime}(\mathrm{E})\left(f^{\prime \prime}(\mathrm{E})\right.$ is proportional to $\mathrm{E} \mu(\mathrm{E})$ and to absorption) are not independent, but are related by the Kramers-Kronig transforms.

Variations of the energy dependent scattering factors $f^{\prime}$ and $f^{\prime \prime}$ close to $\mathrm{K}$ edges are generally are quite limited in magnitude. If they are larger for other edges, their photon energy is generally too low to perform powder diffraction measurements over a large enough Q range. As an example for the Sr K edge, $f^{\prime}$ variations do not exceed 5 e.u. (electron units), to be compared with the 37 e.u. of the Thomson scattering. Moreover these values strongly decrease when moving away for the edge: as an example, $f^{\prime}$ equals to $-6.8,-4.9,-2.6 \mathrm{e} . \mathrm{u}$ at 10,65 and $900 \mathrm{eV}$ respectively below the Sr K absorption edge as measured on a powder of Sr-exchanged X zeolite [23]. Consequently intensity variations due to resonant effects are generally small. For powder diffraction analysis at multiple photon energies, the diffracted intensity measurement has therefore to be very accurate and the well known requirements for powder X-ray 


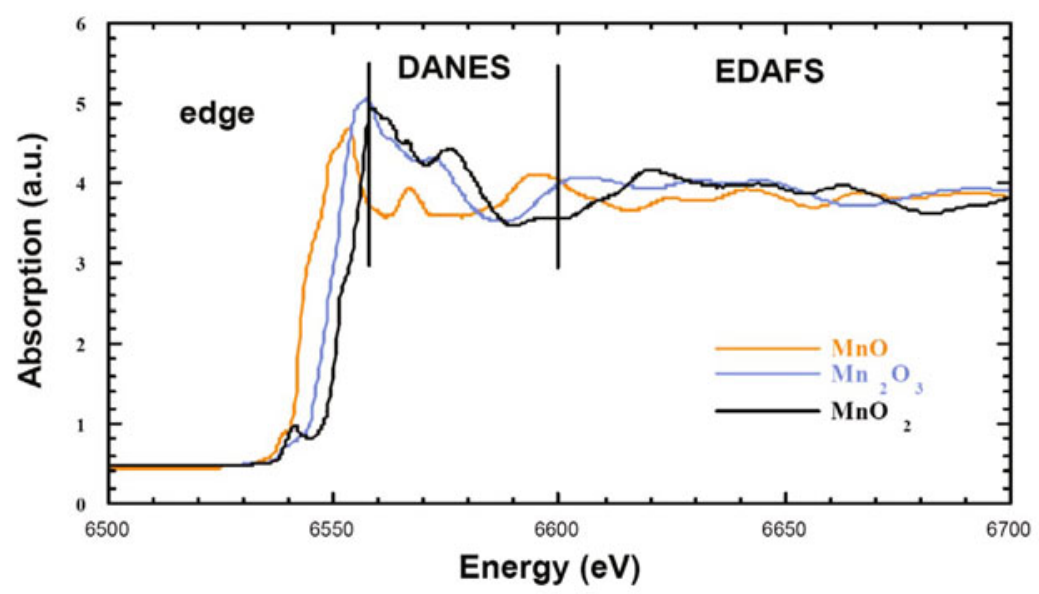

Fig. 1. Influence of $\mathrm{Mn}$ valence state on $\mathrm{Mn}$ absorption $\mathrm{K}$ edges position. Comparison between absorption spectra collected on different Mn oxides [39].

diffraction have to be met: good sampling statistics, appropriate corrections of both texture and absorption.

From a more theoretical point of view, diffraction on a powder is affected by three main limitations (compared to single crystal X-ray diffraction): (i) Bragg reflection overlap, (ii) lower intensity range (iii) higher background. There is also the problem that Friedel reflections (hkl) and $(-h-k-l)$ obviously overlap and even on-resonance powder X-ray diffraction analysis is nearly insensitive to $f^{\prime \prime}$ variations.

\section{Valence and crystallographic environment selectivity}

As the real and imaginary factors $f^{\prime}$ and $f^{\prime \prime}$ are related to absorption, they exhibit the same sensitivities to valence states and crystallographic environment as does X-ray absorption. Fig. 1 shows for illustration the energy shifts obtained between spectra measured on different oxides at the Mn K absorption edge. Thus, resonant scattering can be used not only to characterize chemical contrast, but also to analyze both valence states and crystallographic environments.

In this section, resonant diffraction studies referred as to DAFS (Diffraction Anomalous Fine Structure) are separated into two classes, DANES (Diffraction Anomalous Near Edge Spectroscopy) and EDAFS (Extended Diffraction Anomalous Fine Structure) which consider the energy range close and far from the edge respectively (cf. Fig. 1).

\subsection{DANES: Mixed valence powders}

For resonant scattering measurements, the long range structural information contained in the diffraction peaks is combined with the chemical and local structure selectivity of XANES (X-ray Absorption Near Edge Spectroscopy). This means that DANES experiments are useful to determine the valence of a given element in different crystallographic sites of the same structure or to examine site distortion due to anisotropy of anomalous scattering that makes anomalous sites non-equivalent 
$[22,24,25]$. This last effect can be easily seen by studying weak superstructure reflections and on forbidden reflections in single crystals or thin films [22,26-28]. With powders, both the worst signal to noise ratio and the partial averaging of the anisotropy of anomalous scattering make this kind of analysis almost impossible. This can be seen as an important limitation of these measurements on powders since these last cases are generally considered as very favorable for investigations by resonant scattering. Indeed the diffracted signal is only made of resonant contributions and relative absorption corrections are the weakest.

From a methodological point of view, the main scheme is to first refine independently $f^{\prime}$ values of the resonant atoms at a few energies and to interpret in a second step their differences in terms of valences. However some care must be taken since the $f^{\prime}$ dependence with energy, close to the absorption edge, is also related to the environment of the resonant element and to its anisotropy (as for XANES). Many studies have been performed on powders to investigate this issue such as for example, during the "high $\mathrm{T}_{\mathrm{c}}$ superconductor research rush", to study the copper valence state for the two different $\mathrm{Cu}$ sites in $\mathrm{YBa}_{2} \mathrm{Cu}_{3} \mathrm{O}_{7}$ and $\mathrm{YBa}_{2} \mathrm{Cu}_{3} \mathrm{O}_{6}$ compounds [29], and for $\mathrm{Eu}_{3} \mathrm{O}_{4}$ oxide to localize the $\mathrm{Eu}^{3+}$ and $\mathrm{Eu}^{2+}$ cations in the three different sites [30], as well as to analyze the $\mathrm{Ga}^{+} \mathrm{Ga}^{3+} \mathrm{Cl}_{4}$ mixed-valence oxide [31]. These valence studies can be associated with different cation occupancies as it was done with a mineralogy compound to determine the Mn valence state and its sites occupancy namely as in $\mathrm{Ca}-(\mathrm{Al}, \mathrm{Mn}, \mathrm{Mg}, \mathrm{Fe}) \mathrm{Si}-\mathrm{O}$ pumpellyite [32].

In magnetite, one has to admit that most significant advances on the understanding of charge ordering at low temperature have been achieved using resonant diffraction on single crystals [28] Lorenzo et al., Herrero-Martin et al. in this special issue. However it must be mentioned that Goff et al. [33] also succeeded in obtaining evidence about such a charge ordering with resonant diffraction on a polycrystalline sample. They refined the values of $f^{\prime}$ and $f^{\prime \prime}$ relating to Fe elements using full diffraction patterns measured at three energies. In this last study, even if Fe resonant effects are quite weak (as observed on the overall profile fitting agreement factors), Fe charge order induces significant differences for some $(h k l)$ reflections. For even more complex ferrites like $\left(\mathrm{Fe}_{2.75} \mathrm{Ti}_{0.25}\right)_{1-\delta} \mathrm{O}_{4}$, the study of cation sites occupancy (by Fe ${ }^{2+}, \mathrm{Fe}^{3+}$, $\mathrm{Ti}^{4+}$ and vacancies) could not be performed using this methodology [34]: its limits for powder samples had been reached. As a consequence the authors were compelled to use $f^{\prime}$ and $f^{\prime \prime}$ for Fe elements measured on reference samples, their refinement not being possible.

Another approach is to collect diffraction data versus energies on few different Bragg lines. This strategy has been initiated by Attfield [35] to probe the copper oxidation state distribution in $\mathrm{YBa}_{2} \mathrm{Cu}_{3} \mathrm{O}_{6.3}$. The same method was used on the $\mathrm{Co}_{3} \mathrm{O}_{4}$ compound by Pickering et al. to extract site selective spectra of Co [36,37]. However DANES data collection is synchrotron beam time consuming, since it implies the measurement of many reflections at numerous energies, and methodological studies have largely been performed, rather than a wide range of samples. Different procedures for characterizing $\left(\mathrm{Bi}_{1.67} \mathrm{~Pb}_{0.33}\right) \mathrm{PtO}_{4}$ powders by this technique were explored by Vacinova et al. [38]. Experiments have been performed at the $\mathrm{Pt} \mathrm{L}_{\mathrm{III}}$ edge $(11564 \mathrm{eV})$, but also at high energy at the Pt $\mathrm{K}$ edge $(78395 \mathrm{eV})$, using several data collection setups (comprising analyzer crystals, 1D-detector, 2D-detector). The authors compared these experimental procedures and analyses and showed that DANES experiments with a 2D-detector are possible. All these studies have shown the possibility, but also the intrinsic limits, of the DANES method applied to powders namely a low count rate, as well as problems associated with crystallites sampling and fluorescence scattering. 


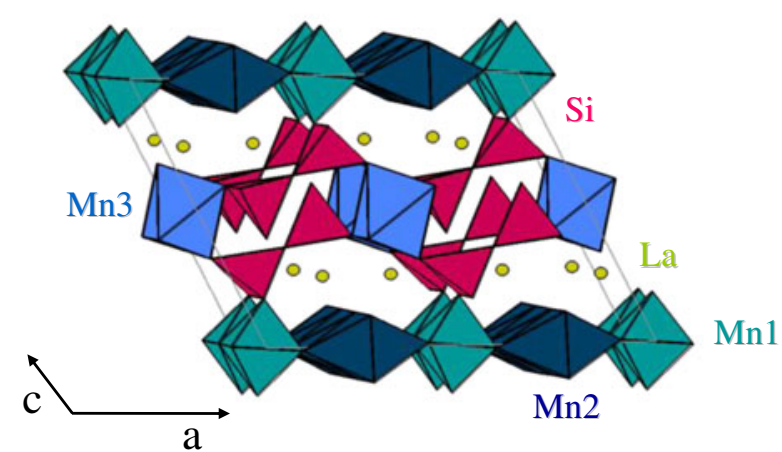

Fig. 2. Definition of the three different $\mathrm{Mn}$ sites in $\mathrm{La}_{4} \mathrm{Mn}_{5} \mathrm{Si}_{4} \mathrm{O}_{22}$ crystal structure. Oxygen atoms are located at the vertices of octahedrons (around the Mn cations with different valence states) and tetrahedrons (around Si atoms) [39].

A second methodological work on DANES has been undertaken on a case study example: the highly-absorbing mixed-valence manganese oxide $\mathrm{La}_{4} \mathrm{Mn}_{5} \mathrm{Si}_{4} \mathrm{O}_{22}$. The work presented in the following is described in much more details elsewhere [39]. This X-ray absorbing compound (X-ray absorption length of about 8 microns at energies lower than the Mn K absorption edge) contains three valence states of manganese, namely $\mathrm{Mn}^{4+}, \mathrm{Mn}^{3+}$ and $\mathrm{Mn}^{2+}$, distributed over three distinct crystallographic sites (cf. Fig. 2): previous studies have suggested the presence of a single valence state in each manganese site [40].

Diffracted intensities were collected around the $\mathrm{K}$ absorption edge of manganese $(6539 \mathrm{eV})$ at the ESRF on the BM2 beamline [41]. Regarding data collection, energy scans at fixed $Q$ positions (on selected reflections) were preferred to the collection of full powder patterns at a discrete but relatively large (20) number of energies. Because of the high powder absorption at the low energies of interest, crystallites sampling is bad. The X-ray beam probes only a few microns in depth and consequently only a limited number of powders grains may diffract. The grain size distribution is greatly enhanced by both spinning the sample and by using analyzers with a large angular acceptance: the mosaïcity of the crystal grains has to be optimized to efficiently remove most of the fluorescence signal [42]. Under these conditions very good intensity versus energy spectra have been obtained for different reflections: they can be used for DANES analysis (cf. Fig. 3).

The strongest contribution to the variation of the diffracted intensities comes from the X-ray absorption, thus this effect must be corrected to a high degree of accuracy. In this case, the absorption coefficient was extracted from the fluorescence spectra collected simultaneously with the diffracted intensities. DANES analysis is possible and was performed by using eight reflections containing the contributions of the three manganese sites (cf. Fig. 4). Normalization of the calculated intensities was done by adjusting the observed data far from the edge to the theoretical $f^{\prime}$ and $f^{\prime \prime}$ values Then a refinement of the resonant scattering factors for the three different manganese sites of this structure has been performed: it gave three different edge positions (cf. Fig. 5). They were shifted by 3 and $4 \mathrm{eV}$ respectively. This quantitative analysis demonstrates that $\mathrm{Mn}$ elements occupying the three different non-equivalent sites in $\mathrm{La}_{4} \mathrm{Mn}_{5} \mathrm{Si}_{4} \mathrm{O}_{22}$ can exhibit different oxidation states. To conclude, this example shows that valence states and DANES studies can also be performed on powdered samples even in challenging cases involving for example high absorption. 

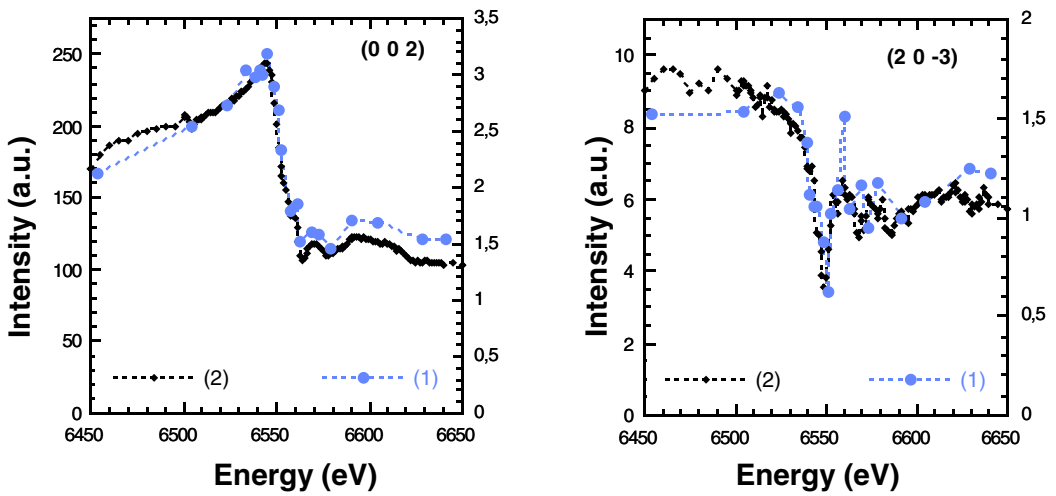

Fig. 3. Influence of data collection strategy on the quality of measured DANES spectra. Strategy 1 refers to the measurement of full $2 \theta$ powder patterns (many Bragg lines) at a few energies (20) when strategy 2 is based on the acquisition of a few reflections (8) at many energies (140). The illustration is for the (002) and (20-3) Bragg lines measured on a $\mathrm{La}_{4} \mathrm{Mn}_{5} \mathrm{Si}_{4} \mathrm{O}_{22}$ powder sample at the Mn K edge [39].
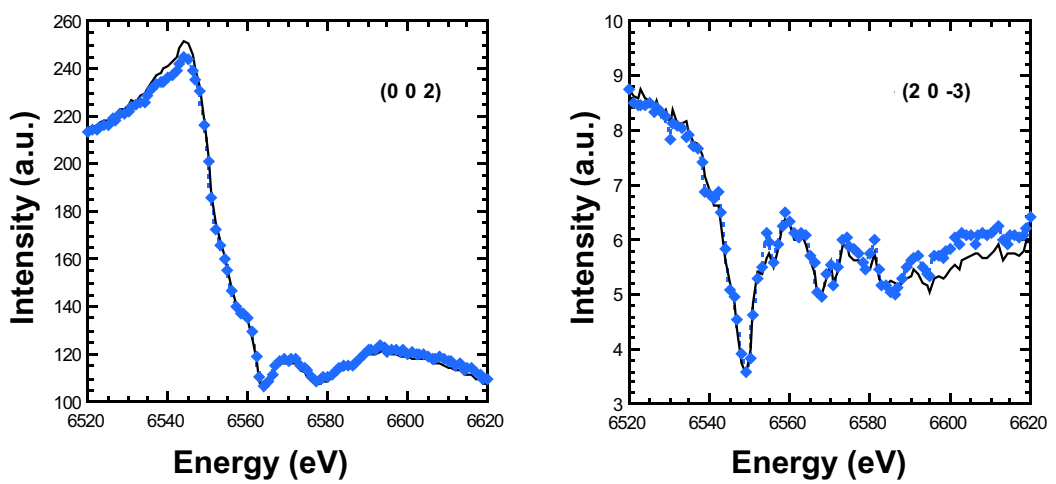

Fig. 4. Good agreement between measured (dots) and calculated (solid lines) for two DANES spectra ((002) and (20-3) reflections). Data were collected on a $\mathrm{La}_{4} \mathrm{Mn}_{5} \mathrm{Si}_{4} \mathrm{O}_{22}$ powder sample at the Mn K edge [39].
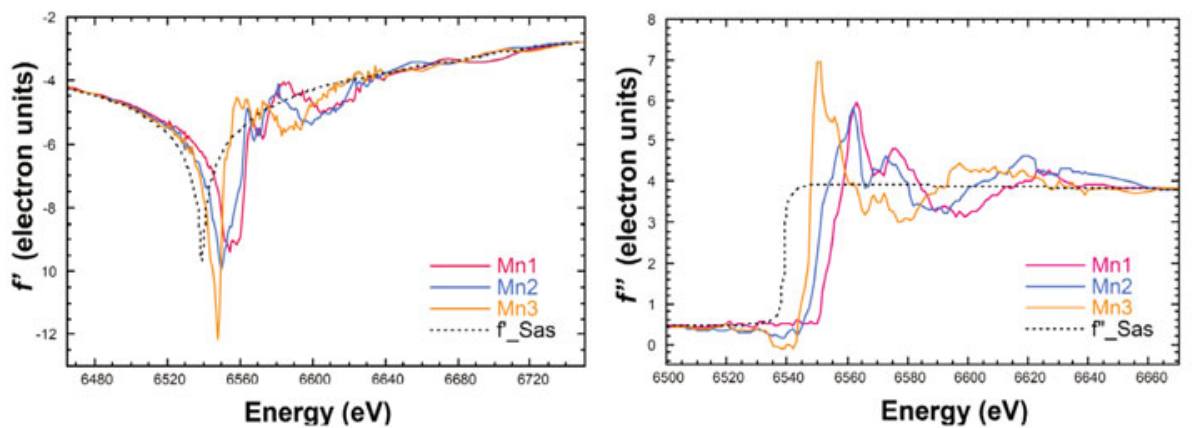

Fig. 5. Refined $f^{\prime}$ and $f^{\prime \prime}$ curves for the different Mn sites with diffraction data measured in the vicinity of the $\mathrm{Mn} \mathrm{K}$ edge on $\mathrm{La}_{4} \mathrm{Mn}_{5} \mathrm{Si}_{4} \mathrm{O}_{22}$. The occurrence of the different valence states of manganese is demonstrated [39]. 


\subsection{EDAFS for site selective studies}

Contrary to XAFS, DAFS spectroscopy is site-selective because X-ray scattering is also measured $[16,43]$. However to quantitatively analyze EDAFS oscillations, EDAFS patterns must be of very high quality i.e. fulfill the same requirements as those needed for interpreting XAFS data. This means that the diffracted intensity (as a function of the energy) must be measured with a high signal-to-noise ratio (at least 1000) and this, without any systematic errors. Thus these experiments require both an adapted instrumentation for limiting and monitoring the fluctuations of the incoming X-ray beam intensity (normalization) and, more challenging, remaining in perfect powder sampling conditions during the whole energy scan. This last difficulty together with lower statistics associated with powder diffraction (compared to single crystal X-ray diffraction), have strongly limited the applications of EDAFS to powder characterizations. For this reason, resonant scattering studies on powders are usually focused on DANES measurements rather on EDAFS (cf. Fig. 1).

Nevertheless a very convincing pioneering work based on EDAFS experiments on powders had been published quite early (1993). It was dedicated to the analysis of $\mathrm{Co}$ in $\mathrm{Co}_{3} \mathrm{O}_{4}$. These authors succeeded in extracting site selective spectra of Co in the octahedral and tetrahedral sites by using site-selective reflections [36, 37]. More recently, EDAFS has also been used to characterize the Fe location in a strongly distorted $\mathrm{Fe} / \mathrm{FeOx}$ core/shell system. These measurements have been found to nicely complement pure XAFS and X-ray powder diffraction analyses. Moreover the authors mentioned the potentialities of the EDAFS technique for studying nanocrystalline powders because in this specific case, the sampling problem is avoided [44].

However the EDAFS technique (combined or not with DANES) seem to be far more powerful when applied to thin films, to determine the local environment of $\mathrm{Cu}$ sites in the superconductor $\mathrm{YBa}_{2} \mathrm{Cu}_{3} \mathrm{O}_{7}[45,46]$ or to investigate the interface structure of an Ir/Fe superlattice [47].

\section{Element selective contrast studies}

The easiest and most common application of resonant powder diffraction deals definitely with contrast analyses. This technique has been used to discriminate site occupancies of atoms with a close number of electrons in oxides [19,48], in zinc ferrites particles [11], in InSb at high-pressures [49] and also in $\mathrm{Pb}_{5} \mathrm{Bi}_{6} \mathrm{Se}_{14}$ to determine metal ion distributions [50]. Note that in this last case, Zhang and Wilkinson have used resonant scattering at very high energy $(\mathrm{Bi} / \mathrm{Pb} K$-edges). In addition to case study samples, this methods has been used for characterising applied materials such as for battery electrodes (i.e. $\mathrm{La}\left(\mathrm{Ni}_{4-x} \mathrm{Mn}_{x}\right)\left(\mathrm{Al}_{1-y} \mathrm{Co}_{y}\right)$ ) [51], magnetite and complex ferrites [52] and zeolites [53-55].

For complex mixed occupancy compounds, to increase the chemical contrast selectivity, resonant diffraction measurements can be performed on the same sample at the edges of the different elements as in doped high $\mathrm{T}_{\mathrm{c}}$ superconductors or in ternary alloys $[13,19,56-58]$.

It is also possible to use both neutron data and resonant X-ray experiments in combination as for the case of the localization of cations in the non-stoichiometric $\mathrm{La}\left(\mathrm{Ni}_{1-x} \mathrm{Cu}_{x}\right)_{5+\delta}$ compound [59] or for solving for the neighboring-elements in $\mathrm{Sr}_{8} \mathrm{Ga}_{16} \mathrm{Ge}_{30}$ [60]. In earlier reviews, resonant XRPD and neutron diffraction techniques have been described and compared [17,61,62]. Neutron diffraction has three important advantages: (i) the possibility to characterise light element atoms, (ii) the possibility to get high quality data even at very high $\mathrm{Q}$ values and (iii) contrast enhancement by isotopic exchange. A comparative study of resonant X-ray diffraction 
and neutron diffraction for contrast analyses has been reported by Warner et al. [63] for quantification of iron/cobalt ordering over different sites in $\mathrm{Co}_{2} \mathrm{Fe}\left(\mathrm{PO}_{4}\right)_{2}$. These authors concluded that both techniques provided close $\mathrm{Co} / \mathrm{Fe}$ occupancy values and with similar precisions. It seems that no general rule on the efficiency of one technique over the other can be given, however one interest of neutron diffraction is that the isotopic scattering power can be exploited and thereby present large variations, and this contrast can be important for a compound with an isotope having a negative neutron scattering factor.

In the next section both data collection and refinement strategies for contrast analyses will be discussed. Then an example will be highlighted namely the cation distribution in $\mathrm{X} / \mathrm{Y}$ zeolites under different adsorption (water, xylene) conditions.

\subsection{Data collection \& refinement strategies}

The most commonly used methodology for performing X-ray contrast studies is to collect at least one diffraction pattern at a photon energy slightly below an X-ray absorption edge of the element of interest and another one at an energy far below this absorption edge. Therefore in these two diffraction patterns, the intensity differences come only from the $f^{\prime}$ evolution of the "resonant scatterer". Furthermore measurements at energies above the absorption edge exhibit an intrinsically worse signal to noise ratio (because of the fluorescence), even if advanced detection systems may be used (a crystal analyser with an enlarged mosaïcity) to overcome this problem.

Concerning the angular range to characterize, Lorimier et al. stress the importance of measuring high $\mathrm{Q}$ values for at least one powder pattern [34]. Some authors have measured more than two energies for a given absorption edge (see for example [23]), but the interest of such a redundancy is not straightforward and seems to be sample dependent. Moreover it has been seen for a long time as a time-consuming strategy incompatible with in-situ measurements or just with synchrotron beam time being limited. With the availability of $1 \mathrm{D}$ pixel detectors [64] and 2D detectors [50,65], limited beamtime cannot really be seen anymore as a limit.

However the measurement of powder diffraction patterns at the absorption edges of as many of the elements in a given compound as possible is a fruitful choice $[60,66,67]$. Finally one has to keep in mind that contrary to DANES measurements (see section 3.a), $f^{\prime}$ values are generally not refined in contrast analyses, but measured through the acquisition of fluorescence spectra on the sample itself and a subsequent Kramers-Kronig transformation calculation. Using these measured/derived $f^{\prime}$ values, the different powder patterns can be refined simultaneously. Many diffraction programs can be used for these analyses (FullProf [68], GSAS [69], XND [70], JANA [71]) where $f^{\prime}$ and $f^{\prime \prime}$ values for each scatterer may be defined manually.

The influence of data refinement methodology on the resulting site occupancy has been analysed in depth on powders [60] and in single crystals [72]. On powders, the conclusions are that constraints on the site occupancy have to be used. This may be explained either by systematic errors or/and the low signal to noise ratio (associated with powder diffraction). Based on these conclusions and to optimise the data refinement strategy for resonant powder diffraction, two alternatives have been proposed [67]: (i) dispersive difference maps as commonly used for single crystal X-ray diffraction (coming from macromolecular crystallography and already applied in materials science $[56,72,73]$ ) (ii) dispersive difference patterns as suggested earlier [74]. These two processes have been developed to better differentiate non resonant elements from the resonant ones. Note that for these calculations based on differences between data obtained at two energies, the ideal case is to identify Bragg lines for which anomalous scatterers do not contribute: thus intensities in diagrams collected at very 
(a)

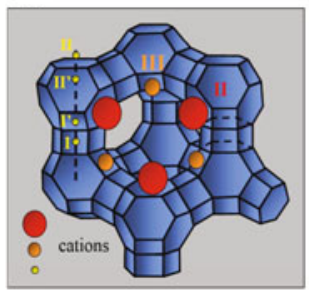

(b)

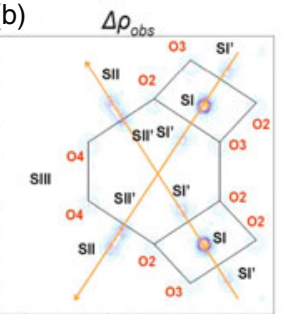

(c) $\Delta \rho_{\text {obs }}$ at $\mathrm{Sr} K$ edge

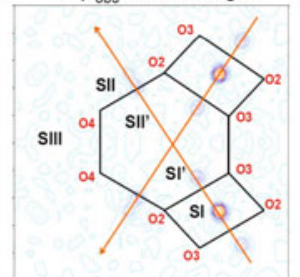

(d) $\Delta \rho_{\text {obs }}$ at $R b$ K edge

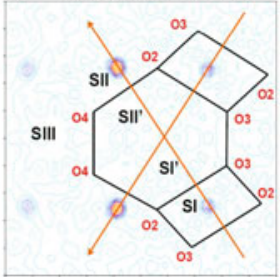

Fig. 6. The X zeolite structure: (a) the main cationic sites in the unit cell, (b) the intersection of two $\langle 111\rangle$ axes defines the Fourier map section, (c) Dispersive difference Fourier map for $\mathrm{Rb}$, (d) Dispersive difference Fourier map for Sr.

different energies can be normalized. This methodology is detailed in the following section.

\subsection{The case of bicationic zeolites under a multi-component adsorbed phase}

High performance industrial processes for separation and purification of hydrocarbon isomers are based on adsorption properties of molecular sieves such as $\mathrm{X}, \mathrm{Y}$ and A zeolites containing cations like $\mathrm{Na}^{+}, \mathrm{Ba}^{2+}, \mathrm{K}^{+}, \mathrm{Ca}^{2+}, \mathrm{Rb}^{+}$. These cations can occupy different sites in larges zeolite cavities, in $\mathrm{X}$ zeolites as an example, four main crystallographic sites can be occupied by cations (cf. Fig. 6a). The occupancy factor of each site depends on the water content of the zeolite. In hydrated zeolites, cations are mainly co-ordinated by water molecules and sit preferentially in open sites of the framework. Upon dehydration, they move to more confined sites where they can achieve full co-ordination with the framework oxygen [75]. The adsorption properties of the zeolite can be improved by the presence of two different cations and the distribution of one type of cation, among the zeolite framework sites as a function of dehydration, is modified by the presence of the second cation. Such information is important since there is a high level of correlation between the location of the adsorbed molecules in the zeolite pores and the position of the zeolite cations. Thus the size and the electronic properties of the cation can play a special role in the selectivity of the adsorbent towards the different isomers.

Resonant scattering is essential to study interactions when there are two or more cations. This has been done in the field of catalysis on $\mathrm{Ag}-\mathrm{Y}$ Zeolite to localize $\mathrm{Ag}^{+}$ [53], on the case of multi-cations exchanged X or Y Zeolite to follow the evolution of total cationic site occupancy and cationic distribution under in situ conditions [23]. The habits of different couples of cations were studied as a function of their size and electric charge $(\mathrm{Na}, \mathrm{K}, \mathrm{Rb}, \mathrm{Cs}, \mathrm{Sr}, \mathrm{Ba}, \ldots$ ) and analyzed in operating e.g. chemical catalysis conditions. Herein, we focus on the $\mathrm{Sr}$ and $\mathrm{Rb}$ case since these cations are close elements in the periodic table and have nearly the same scattering power either using $\mathrm{X}$ rays or neutrons. $\mathrm{Sr}^{2+}$ and $\mathrm{Rb}^{+}$have the same number of electrons $(\mathrm{Z}=35$ e.u. $)$ and nearly identical neutron scattering lengths $\left(\mathrm{b}(\mathrm{Sr})=7.02 \times 10^{-15} \mathrm{~m}\right.$; $\left.\mathrm{b}(\mathrm{Rb})=7.09 \times 10^{-15} \mathrm{~m}\right)$, so this is a particularly difficult case for conventional X-ray or neutron diffraction and exceptionally well suited to REXS methods.

For this last study, resonant contrast diffraction was applied via a methodology which uses (i) dispersive difference maps to directly visualize the resonant scatterers and (ii) simultaneous refinement of several resonant diffraction patterns. The experimental and computational approaches were validated by the determination $\mathrm{Sr}^{2+}$ and $\mathrm{Rb}^{+}$cation distributions in SrRbX [67]. Diffraction experiments (Debye-Scherrer 
(a)

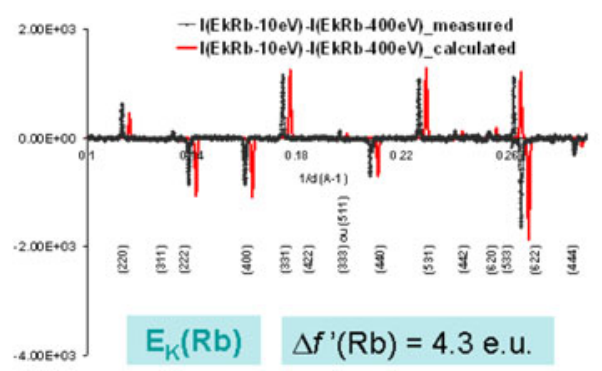

(b)

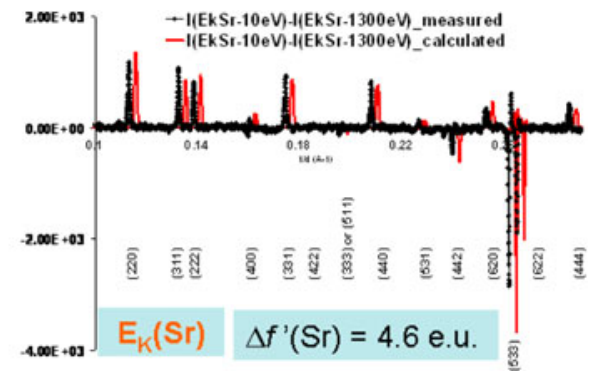

Fig. 7. Measured and calculated with a $1 /$ d shift anomalous differential patterns (difference between powder diffraction diagrams obtained at different energies) at the Rb- (a) and the Sr- (b) K absorption edges, using the same intensity normalization.

geometry) were performed $10 \mathrm{eV}$ below the $\mathrm{Rb}(15202 \mathrm{eV})$ and the $\mathrm{Sr} \mathrm{K}(16105 \mathrm{eV})$ edges and at $14800 \mathrm{eV}$, i.e. far below both edges. An initial Fourier map calculated using the known zeolite structure (cf. Fig. 6a), gave rough and non-element-selective localization of extra-framework atoms (cations, water molecules, etc). These were introduced as electron densities, with no assumption of the atom type, on sites I, I', II, II' and III (cf. Fig. 6b). These resonant diffraction measurements also yielded dispersive difference maps for the electron densities determined at the $\mathrm{Rb}$ and $\mathrm{Sr}$ edges, with amplitudes proportional to the variation of the real resonant contribution $\left(\Delta f^{\prime}\right)$, readily allowing resonant cation localization (cf. Fig. 6c and Fig. 6d).

This chemical selectivity can be seen directly in the anomalous difference patterns calculated from data at $\mathrm{E}_{\mathrm{K}}(\mathrm{Sr})-10 \mathrm{eV}$ minus $\mathrm{E}_{\mathrm{K}}(\mathrm{Sr})-1300 \mathrm{eV}$ (and at $\mathrm{E}_{\mathrm{K}}(\mathrm{Rb})-$ $10 \mathrm{eV}$ minus $\left.\mathrm{E}_{\mathrm{K}}(\mathrm{Rb})-400 \mathrm{eV}\right)$. Calculated and measured patterns, highly-sensitive to resonant atom location and occupancy, gave close agreement after adjusting resonant atom occupancies (cf. Fig. 7). Using such a resonant contrast method, the cation distribution was determined in several bicationic zeolites ( $\mathrm{SrRbX}, \mathrm{SrCaX}$ and $\mathrm{CaRbX}) \cdot \mathrm{Sr}^{2+}$ occupies preferentially sites I', II and III whereas $\mathrm{Rb}^{+}$occupies mainly site II and if enough $\mathrm{Rb}^{+}$is present super-cage site III. $\mathrm{Ca}^{2+}$ shows a strong preference for sites I' and III. In all solids, site II' is mainly populated by water molecules.

In conclusion, resonant scattering enables accurate localization of atoms in powders via dispersive difference maps in real space to locate resonant cations, and via resonant differential powder diffraction patterns in reciprocal space to quantify the structural model. Using this new methodology, resonant contrast diffraction becomes an easily applicable key method to analyze site occupancies of chemically different cations, even in the presence of other scatterers like organic ones, and can also be performed under in operando conditions as it was done on $\mathrm{SrRbX}$ with water or a para-xylene flux (cf. Fig. 8) [23].

\section{Structure solution and MAD on macromolecular powder}

Since its demonstration on polycrystalline materials [76-79], almost no application of resonant diffraction has been proposed for de novo structure determination of small molecules. This is probably related to the efficiency of conventional crystallography methods for elucidating those crystal structures. The most striking example is very probably given by the work of Burger et al. [80] which combined MEM (Maximun Entropy Methods) and MAD methods for the de novo structure solution from 


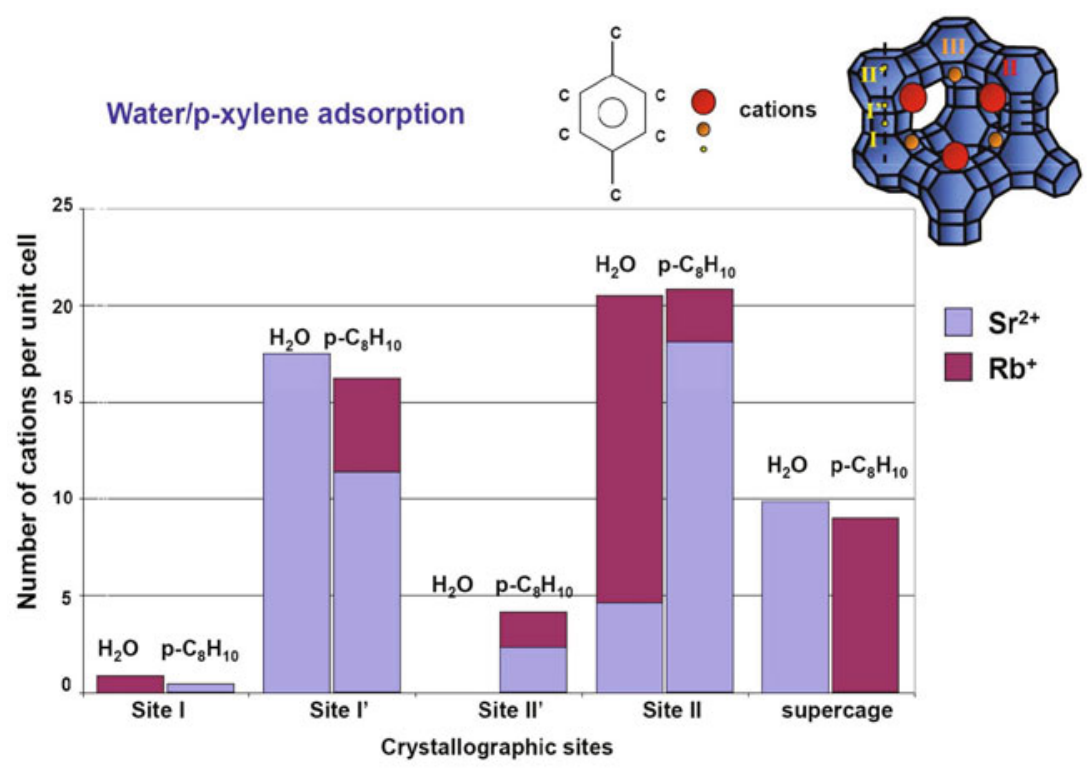

Fig. 8. Example of $\mathrm{Sr}$ and Rb localization in X-zeolite cage-sites during in operando conditions with adsorption of water or para-xylene.

powder data measured on the $\mathrm{SrSO}_{4}$ compound. By combining of three resonant powder patterns (measured at Sr K edge), partial Patterson density maps (describing the Sr-Sr pairs) were calculated to locate the $\mathrm{Sr}$ resonant cations and then used to phase the non-overlapping reflections. Note that contrary to the Fourier map, calculated MEM density maps clearly showed all the light oxygen atoms and enabled a complete solution for the whole structure.

An important application of MAD techniques has been recently undertaken on powders for solving the structure of single crystals of biological macromolecules [8183]. As an example of seeking to apply such an approach to protein powders, recently Helliwell et al. [84] recorded high quality synchrotron X-ray protein powder diffractograms (ID31, ESRF) from $\mathrm{K}_{2} \mathrm{PtBr}_{6}$ bound to lysozyme at $80 \mathrm{~K}$ to protect against $\mathrm{X}$-radiation damage as much as possible and also to trap i.e. fix the $\mathrm{K}_{2} \mathrm{PtBr}_{6}$ heavy atom compound bound state. With multiple powder pattern analysis i.e. taking advantage of the anisotropic variation of unit cell parameters with the steadily increasing X-ray dose, they extracted individual reflection intensities and thus were able to show the presence of $\mathrm{PtBr}_{6}^{2-}$ bound in lysozyme in $\left(\mathrm{F}_{\mathrm{o}}-\mathrm{F}_{\mathrm{c}}\right)$ Fourier maps at two binding sites. The resonant dispersive difference Patterson at two energies around the $\mathrm{Br} \mathrm{K}$ edge and also the Pt $\mathrm{L}_{\mathrm{III}}$ edge showed up binding site1 in one of the six samples tested. In order to investigate the detailed chemical binding behaviour, single crystal analyses were conducted with time-resolved freeze quenching after lysozyme single crystal soak times of 10, 90 and 170 minutes. Whilst the quick soaking of 10 minutes, used in first powder experiments, showed clear binding, there was a steady progression of increasing binding strength with increasing soak time evident in these further single crystal characterizations. Thus, such a time-resolved analytical chemistry analysis showed that further heavy atom signal optimizations, and reproducible behaviour, are possible. Recent experiments for extending such an approach to the yet larger isomorphous and energy dispersive signal cases of $\mathrm{Ta}_{6} \mathrm{Br}_{12}$ as well as the non-ionic Gadolinium chelate $\mathrm{Gd}(\mathrm{HP}-\mathrm{D} 03 \mathrm{~A})\left(\mathrm{C}_{17} \mathrm{H}_{29}-\mathrm{N}_{4} 0_{7} \mathrm{Gd} \cdot 1.3 \mathrm{H}_{2} 0\right)$ bound to lysozyme for powder experiments have been performed and data analysis is currently in progress. 
Overall, such multi heavy atom cluster compounds like $\mathrm{K}_{2} \mathrm{PtBr}_{6}$ and $\mathrm{Ta}_{6} \mathrm{Br}_{12}$ offer a way forward to attempt to solve de novo protein structures by powder diffraction as a complement to micro-crystallography or poly-crystal crystallography $[85,86]$.

\section{Conclusion}

Over the years, resonant synchrotron X-ray diffraction has become a robust technique even for powders to investigate the valence or the occupancy of given elements in a structure. Moreover the limitations (compared to single crystals) associated with the characterization of powders, are now well known and can be better controlled. Taking advantage of the current advances in detection (pixel detectors), resonant diffraction for example is now adapted for time resolved studies and can be routinely applied for in-situ measurements in chemistry [23]. It is quite surprising that almost no resonant diffraction study has been performed on mixed phase powders: this method would be useful to analyse quantitatively the "real materials" such as ores. Finally, it is believed that the future of this technique could also be linked to macromolecular crystallography [17]. Indeed several advances in that field have been performed in the recent year by powder diffraction, and adapting the use of MAD to protein powders could be a useful adjunct to help solve ab-initio macromolecular structures.

J-L. H. and J-F. B. are grateful to IFP-Energies Nouvelles for funding of the H.P Ph.D thesis and would like to thank C. Pichon, B. Rebours and J. Lynch for their collaboration and referees for their comments and suggestions. The authors have been deeply affected by the too early death of Dr. J. Lynch (IFP-Energies Nouvelles) in 2010.

\section{References}

1. D. Coster, K.S. Knol, J.A. Prins, Z. Phys. 63, 345 (1930)

2. H. Mark, L.Z. Szilaid, Z. Phys. 33, 688 (1925)

3. A.J. Bradley, J.W. Rodgers, Proc. Roy. Soc. A 144, 340 (1934)

4. F.W. Jones, C. Sykes, Proc. Roy. Soc. A 161, 440 (1937)

5. J.M. Bijvoet, Proc. Acad. Sci. Amsterdam B 52, 313 (1949)

6. R. Fourme, W. Shepard, M. Schiltz, M. Ramin, R. Kahn in HERCULES, vol. IV, edited by E. Fanchon, E. Geissler, J.-L. Hodeau, J-R. Regnard, P. Timmins (Oxford Univ. Press, Oxford, 2000), p. 36.

7. W.A. Hendrickson, Science 254, 51 (1991)

8. J.R. Helliwell, in Macromolecular Crystallography with Synchrotron Radiation (Cambridge University Press, London, 1992)

9. G. Materlink, C.J. Sparks, K. Fischer, Resonant Anomalous X-ray Scattering (Elsevier Science B.V., Amsterdam, 1994)

10. G.E. Ice, C.J. Sparks, in Resonant Anomalous X-ray Scattering, edited by G. Materlink, C.J. Sparks, K. Fischer (Elsevier Science B.V., Amsterdam, 1994), p. 265

11. E. Matsubara, Y. Waseda, in Resonant Anomalous X-ray Scattering, edited by G. Materlink, C.J. Sparks, K. Fischer (Elsevier Science B.V., Amsterdam, 1994), p. 345

12. D. Raoux, in Resonant Anomalous X-ray Scattering, edited by G. Materlink, C.J. Sparks, K. Fischer (Elsevier Science B.V., Amsterdam, 1994), p. 323

13. R.S. Howland, T.H. Geballe, S.S. Laderman, A. Fisher-Colbrie, M. Scott, J.M. Tarascon, P. Barboux, Phys Rev. B 39, 9017 (1989)

14. A.P. Wilkinson, A.K. Cheetham, S.C. Tang, W.J. Reppart, J. Chem. Soc. Chem. Commun. 1485 (1992)

15. D.E. Cox, A.P. Wilkinson, in Resonant Anomalous X-ray Scattering, edited by G. Materlink, C.J. Sparks, K. Fischer (Elsevier Science B.V., Amsterdam, 1994), p. 195 
16. J.L. Hodeau, V. Favre-Nicolin, S. Bos, H. Renevier, E. Lorenzo, J.F. Berar, Chem. Rev. 101, 1843 (2001)

17. M. Cianci, J. R. Helliwell, M. Helliwell, V. Kaucic, N.Z. Logar, G. Mali, N.N. Tusar, Crystallogr. Rev. 11, 245 (2005)

18. Y. Waseda, in Anomalous X-ray Scattering for Materials Characterisation: Atomic-Scale Structure Determination, Springer Tracts in Modern Physics (Springer Verlag, Berlin 2002), p. 179

19. J.P. Attfield, Mater. Sci. Forum 228-231, 201 (1996)

20. D.H. Templeton, L.K. Templeton, Acta Crystallogr. A 36, 436 (1980)

21. V.E. Dmitrienko, Acta Crystallogr. A 39, 29 (1983)

22. A. Kirfel, in Resonant Anomalous X-ray Scattering, edited by G. Materlink, C.J. Sparks, K. Fischer (Elsevier Science B.V., Amsterdam, 1994), p. 231

23. H. Palancher, Ph.D. thesis, Université Joseph Fourier, Grenoble, France, 2004

24. T. Lippmann, A. Kirfel, HASYLAB/DESY Report (1991)

25. W. Morgenroth, A. Kirfel, Z. Kristallogr. 185, 205 (1991)

26. T. Toda, T. Nogami, K. Yamasaki, Y. Soejima, J. Appl. Crystallogr. 31, 423 (1998)

27. J. Garcia, G. Subias, M.G. Proietti, H. Renevier, Y. Joly, J.L. Hodeau, J. Blasco, M.C. Sanchez, J.F. Berar, Phys. Rev. Lett. 85, 578 (2000)

28. Y. Joly, J.E. Lorenzo, E. Nazarenko, J.L. Hodeau, D. Mannix, C. Marin, Phys Rev. B 78, $134110(2008)$

29. G.H. Kwei, R.B. Von Dreele, A. Williams, J.A. Goldstone, A.C. LawsonII, W.K Warburton, J. Mol. structure 223, 383 (1990)

30. J.P. Attfield, Nature 343, 46 (1991)

31. A.P. Wilkinson, A.K. Cheetham, D.E. Cox, Acta Crystallogr. B 47, 155 (1991)

32. G. Artioli, A. Pavese, M. Bellotto, S.P. Collins, G. Lucchetti, Am. Mineral. 81, 603 (1996)

33. R.J. Goff, J.P. Wright, J.P. Attfield, P.G. Radaelli, J. Phys.: Condens. Matter. 17, 7633 (2005)

34. J. Lorimier, F. Bernard, J.-C. Niepce, N. Guigue-Millot, O. Isnard, J.-F. Bérar, J. Appl. Crystallogr. 36, 301 (2003)

35. J.P. Attfield, J. Phys. Chem. Solids 52, 1243 (1991)

36. I.J. Pickering, M. Samsone, J. Marsch, G.N. George, J. Am. Chem. Soc. 115, 6302 (1993)

37. I.J. Pickering, M. Sansone, J. Marsch, G N. George, Jpn. J. Appl. Phys., Suppl. 32, 206 (1993)

38. J. Vacinova, J.L. Hodeau, P. Bordet, M. Anne, D. Cox, A. Fitch, P. Pattison, W. Schweggle, H. Graafsma, A. Kvick, Mater. Sci. Forum 228, 95 (1996)

39. S. Bos, Ph.D. thesis, Université Joseph Fourier, Grenoble, France, 1999

40. C. Gueho, D. Giaquinta, J.L. Mansot, T. Ebel, P. Palvadeau, Chem. Mater. 7, 486 (1995)

41. H. Renevier, S. Grenier, S. Arnaud, J.F. Bérar, B. Caillot, J.L. Hodeau, A. Letoublon, M.G. Proietti, B. Ravel J. Synchrotron Rad. 10, 435 (2003)

42. T. Wessels, Ph.D. thesis, ETH Zurich, Switzerland, 1999

43. L.B. Sorensen, J.O. Cross, M.N. Newville, B. Ravel, J.J. Rehr, H. Stragier, C.E. Bouldin, J.C. Woicik, in Resonant Anomalous X-ray Scattering, edited by G. Materlink, C.J. Sparks, K. Fischer (Elsevier Science B.V., Amsterdam, 1994), p. 389

44. C. Meneghini, F. Boscherini, L. Pasquini, H. Renevier, J. Appl. Crystallogr. 42, 642 (2009)

45. J.O. Cross, M. Newville, L.B. Sorensen, H.J. Stragier, C.E. Bouldin, J.C. Woicik, J. Phys. IV (France) 7, 745 (1997)

46. J.O. Cross., M. Newville, J.J. Rehr, L.B. Sorensen, C.E. Bouldin, G. Watson, T. Gouder, G.H. Lander, M.I. Bell, Phys. Rev. B 58, 11215 (1998)

47. H. Renevier, J.L. Hodeau, P. Wolfers, S. Andrieu, J. Weigelt, R. Frahm Phys. Rev. Lett. 78, 2775 (1997)

48. D.A. Perkins, J.-P. Attfield, J. Chem. Soc., Chem. Comm. 4, 229 (1991) 
49. R.J. Nelmes, P.D. Hatton, M.I. McMahon, R.O. Piltz, J. Grain, R.J. Cernik, G. Bushnellwye, Rev. Sci. Instrum. 63, 1039 (1992)

50. Y. Zhang, A.P. Wilkinson, P.L. Lee, S.D. Shastri, D. Shu, D.-Y. Chung, M.G. Kanatzidis, J. Appl. Crystallogr. 38, 433 (2005)

51. J.-M. Joubert, R. Cerny, M. Latroche, A. Percheron-Guégan, K. Yvon, J. Appl. Crystallogr. 31, 327 (1998)

52. F. Bernard, J. Lorimier, V. Nivoix, N. Millot, P. Perriat, B. Gillot, J.F. Berar, J.C. Niepce, J. Solid State Chem. 141, 105 (1998)

53. C. Lamberti, M. Milaneso, C. Prestino, S. Bordiga, A.N. Fitch, G.L. Marra, ESRF Newsletter 35 (2001)

54. A.P. Wilkinson, A.K. Cheetham, S.C. Tang, W.J. Reppart, J. Chem. Soc. Chem. Commun. 1485 (1992)

55. H. Palancher, C. Pichon, J.L. Hodeau, J.-F. Berar, J. Lynch, B. Rebours, J. RodriguezCarvajal, Zeit. Kristallogr. Suppl. 23, 487 (2006)

56. G.H. Kwei, R.B. Von Dreele, S.W. Cheong, Z. Fisk, J.D. Thompson, Phys. Rev. B 41, 1889 (1990)

57. M.D. Marcos, M.A.G. Aranda, D.C. Sinclair, J.P. Attfield, Physica C 235, 967 (1994)

58. M.A.G. Aranda, D.C. Sinclair, J.P. Attfield, A.P. McKenzie, Phys. Rev. B 51, 12747 (1995)

59. M. Latroche, J.M. Joubert, A. Percheron-Guegan, P.H.L. Notten, J. Solid State Chem. 146, 313 (1999)

60. Y. Zhang, A.P. Wilkinson, G.S. Nolas, P.L. Lee, J.P. Hodges, J. Appl. Crystallogr. 36, $1182(2003)$

61. A.K. Cheetham, A.P. Wilkinson, Angew. Chem. Int. Ed. 31, 1557 (1992)

62. C. Pichon, H. Palancher, J.L. Hodeau, J.F. Bérar, Oil Gas Sci. Technol. 60, 831 (2005)

63. J.K. Warner, A.P. Wilkinson, A.K. Cheetham, D.E. Cox, J. Phys. Chem. Solids 52, $1251(1991)$

64. S. Basolo, J.-F. Berar, N. Boudet, P. Breugnon, B. Caillot, J.C. Clemens, P. Delpierre, B. Dinkespiler, S. Hustache, I. Koudobine, C. Meessen, M. Menouni, C. Mouget, H. Palancher, P. Pangaud, R. Potheau, E. Vigeolas, J. Synchrotron Rad. 14, 151 (2007)

65. H. Ehrenberg, M. Knapp, T. Hartmann, H. Fuess, T. Wroblewski, J. Appl. Crystallogr. 33, 953 (2000)

66. M. Helliwell, J.R. Helliwell, V. Kaucic, N. Zabukovec Logar, L. Barba, E. Busetto, A. Lausi, Acta Crystallogr. B 55, 327 (1999)

67. H. Palancher, J.-L. Hodeau, C. Pichon, J.-F. Bérar, J. Lynch, B. Rebours, J. RodriguezCarvajal, Angew. Chem. Int. Ed. 44, 1725 (2005)

68. J. Rodriguez-Carvajal, Physica B 192, 55 (1993)

69. A.C. Larson, R.B. Von Dreele GSAS, Report No. LAUR 86-748, Los Alamos National Laboratory (New Mexico, USA, 1987)

70. J.-F. Bérar, G. Baldinozzi, IUCr-CPD Newslett. 20, 3 (1998)

71. V. Petricek, M. Dusek, L. Palatinus, JANA2006 Institute of Physics, Praha, Czech Republic (2006)

72. M. Helliwell, J.R. Helliwell, V. Kaucic, N. Zabukovec Logar, S.J. Teat, J.E. Warren, E.J. Dodson, Acta Crystallogr. B 66, 345 (2010)

73. R. Wulf, Acta Crystallogr. A 46, 681 (1990)

74. K. Sugiyama, Y. Waseda, Materials Transactions, JIM 30, 235 (1989)

75. H. Palancher, C. Pichon, B. Rebours, J.-L. Hodeau, J. Lynch, J.-F. Bérar, S. Prévot, G. Conan, C. Bouchard, J. Appl. Crystallogr. 38, 370 (2005)

76. C.M. Mitchell, Acta Crystallogr. 10, 475 (1957)

77. Y. Okaya, R. Pepinsky, Phys. Rev. 103, 1645 (1956)

78. I.G. Wood, L. Nicholls, G. Brown, J. Appl. Crystallogr. 19, 364 (1986)

79. W. Prandl, Acta Crystallogr. A 46, 988 (1990)

80. K. Burger, D. Cox, R. Papoular, W. Prandl, J. Appl. Crystallogr. 31, 789 (1998)

81. J.R. Helliwell, M. Helliwell, R.H. Jones, Acta Crystallogr. A 61, 568 (2005)

82. J.P. Wright, C. Besnard, I. Margiolaki, S. Basso, F. Camus, A.N. Fitch, G.C. Fox, P. Pattison, M. Schiltz, J. Appl. Crystallogr. 41, 329 (2008) 
83. I. Margiolaki, J.P. Wright, Acta Crystallogr. A 64, 169 (2008)

84. J.R. Helliwell, A.M.T. Bell, P. Bryant, S. Fisher, J. Habash, M. Helliwell, I. Margiolaki, K. Surasak, Y. Watier, J. Wright, S. Yalamanchilli, Z. Kristallogr. 225, 570 (2010)

85. B. Hedman, K.O. Hodgson, J.R. Helliwell, R. Liddington, M.Z. Papiz, P.N.A.S. USA 82, 7604 (1985)

86. K.S. Paithankar, H.O. Sørensen, J.P. Wright, S. Schmidt, H.F. Poulsen, E.F. Garman, Acta Crystallogr. D 67, 608 (2011) 